\title{
Fatores desencadeantes de depressão na adolescência: uma revisão integrativa
}

\author{
Triggering factors for depression in adolescence: an integrative review \\ Factores desconectadores de la depresión en la adolescencia: una revisión integrativa
}

Recebido: 02/12/2021 | Revisado: 07/11/2021 | Aceito: 08/12/2021 | Publicado: 15/12/2021

\author{
Jadiane de Jesus da Silva \\ ORCID: https://orcid.org/0000-0002-2147-776X \\ Centro Universitário da Amazônia, Brasil \\ E-mail: jjadiane@gmail.com \\ Maria da Conceição Caetano de Siqueira \\ ORCID: https://orcid.org/0000-0003-1731-4076 \\ Centro Universitário da Amazônia, Brasil \\ E-mail: siqueira_44@hotmail.com
}

\begin{abstract}
Resumo
Nos últimos anos os transtornos mentais vêm atingindo a população em grande escala, se tornando um dos assuntos mais comentados da atualidade, sendo estes caracterizados por um grupo de distúrbios psíquicos que desencadeiam sintomas envolvendo principalmente a emoção, a função cognitiva e a capacidade de controlar comportamentos complexos, que frequentemente cursam para o caminho crônico, dentre essas psicopatologias estão os transtornos depressivos que tem acometido a população de uma forma progressiva. O objetivo deste estudo é identificar os principais fatores desencadeantes de depressão entre adolescentes. Realizou-se uma revisão integrativa da literatura através de pesquisas em bases de dados utilizando estudos completos e disponíveis nas bases de dados científicas que tinham sido publicados no período de 2010 a 2020. Foram selecionados após a leitura exploratória um total de 15 trabalhos que se enquadram aos objetivos da pesquisa. Os principais fatores desencadeantes de depressão entre adolescentes evidenciados foram: conflitos familiares; vulnerabilidade social; gênero e idade; aspectos psicoafetivos e psicossociais. Com base nas pesquisas realizadas foi possível evidenciar que a depressão entre os adolescentes é multifatorial assim como nos adultos, várias situações corroboram para o seu surgimento, porém grande parte dos estudos apontou como principal fator o âmbito familiar, a forma como esses adolescentes são criados, educados e o vínculo que os mesmos têm com os pais.
\end{abstract}

Palavras-chave: Depressão; Adolescentes; Saúde mental.

\begin{abstract}
In recent years, mental disorders have affected the population on a large scale, becoming one of the most talked about issues today, which are characterized by a group of brain disorders that trigger symptoms mainly involving emotion, cognitive function and the ability to control behavior complexes, which often follow the chronic path, among these psychopathologies are depressive disorders that have affected the population in a frightening way. Depression is considered a psychopathology in the adult stage only, and it has been showing a high incidence among adolescents, ranging from $3.3 \%$ to $12.4 \%$, with a predominance in females. Thus, the research aimed to identify the main triggering factors for depression among adolescents found in the studies. An integrative review of previous studies, complete and available in scientific databases that had been published between 2010 and 2020, was carried out. After exploratory reading, a total of 11 files were selected. The main triggering factors among adolescents highlighted were: family relationships (conflicts and disruption); family background, social vulnerability, gender and age; gender and age; psychoaffective and psychosocial aspects. Based on the researches carried out, it was possible to show that depression among adolescents is multifactorial, as well as in adults, that is, several situations corroborate its emergence, but most studies have pointed out that the main factor is the family environment, the way in which these teenagers are raised, educated and the bond they have with their parents.
\end{abstract}

Keywords: Depression; Teenagers; Mental health.

\section{Resumen}

En los últimos años, los trastornos mentales han afectado a la población a gran escala, convirtiéndose en uno de los temas más comentados en la actualidad, los cuales se caracterizan por un grupo de trastornos psicológicos que desencadenan síntomas que involucran principalmente la emoción, la función cognitiva y la capacidad de controlar los complejos conductuales. , que a menudo siguen el camino crónico, entre estas psicopatologías se encuentran los trastornos depresivos que han afectado progresivamente a la población. El objetivo de este estudio es identificar los principales factores desencadenantes de la depresión en adolescentes. Se realizó una revisión integradora de la literatura mediante búsquedas en bases de datos utilizando estudios completos disponibles en bases de datos científicas que habían sido publicados entre 2010 y 2020. Se seleccionaron un total de 15 trabajos que encajaban después de una lectura exploratoria con los objetivos de la investigación. Los principales factores desencadenantes de la depresión entre los adolescentes fueron: conflictos 
familiares; vulnerabilidad social; género y edad; aspectos psicoafectivos y psicosociales. Con base en las investigaciones realizadas, se pudo evidenciar que la depresión entre los adolescentes es multifactorial, así como en los adultos, varias situaciones corroboran su surgimiento, pero la mayoría de los estudios señalaron como factor principal el entorno familiar, la forma en que estos adolescentes son personas criadas, educadas y el vínculo que tienen con sus padres.

Palabras clave: Depresión; Adolescentes; Salud mental.

\section{Introdução}

Nos últimos anos os transtornos mentais vêm atingindo a população em grande escala, se tornando um dos assuntos mais comentados da atualidade. As pessoas acometidas por essas psicopatologias, desenvolvem alterações nas funções psíquicas, o que por sua vez provoca um conjunto de sintomas que podem durar semanas, meses ou perdurar por anos, interferindo significativamente na vida pessoal, social e profissional do indivíduo, (Feitosa et al. 2011).

Os transtornos psiquiátricos são formados por um grupo de distúrbios cerebrais que desencadeiam sintomas envolvendo principalmente a emoção, a função cognitiva e a capacidade de controlar comportamentos complexos. Um dos principais distúrbios, que é considerado crônico e mais recorrente ao longo da vida, é o transtorno depressivo (Vicelli, 2018).

De acordo com o levantamento realizado pelo Brasil (2019) entre os anos de 2015 e 2018, o Sistema Único de Saúde (SUS) registrou um aumento de 52\% nos atendimentos ambulatoriais e de internações relacionada aos transtornos depressivos, evoluindo de 79.654 para 121.341 na adesão de consultas. No Brasil estima-se que 14, 1 milhões de pessoas possuam diagnóstico de depressão, conforme mostra a Pesquisa Nacional de Saúde (PNS) 2013 (Brasil, 2019). Além de um aumento de 18,4\% no número de pessoas com depressão no mundo, segundo a (Oms, 2019).

De acordo com o DSM-5 os transtornos são classificados de acordo com seus sinais e sintomas, seguindo a seguinte classificação: o transtorno disruptivo de desregulação do humor refere à crianças que apresentam irritabilidade persistente e episódios frequentes de descontrole comportamental extremo, este é acrescentado aos transtornos depressivos para crianças até 12 anos. Representando a condição clássica desse grupo o transtorno depressivo maior se caracteriza por episódios distintos de pelo menos duas semanas de duração, embora a maioria dos episódios permaneça por um tempo consideravelmente maior, envolvendo alterações visíveis de afeto, além da cognição, funções neurovegetativas, e remissões inter episódicas. O transtorno depressivo persistente por sua vez se caracteriza pela perturbação do humor contínuo por pelo menos dois anos em adultos e um ano em crianças. O transtorno disfórico pré menstrual este dá início em algum momento após a ovulação e modera poucos dias após a menstruação, causando impacto significativo no funcionamento. Há também aqueles que são caracterizados pelo abuso do uso de substâncias, medicamentos e diversas outras condições médicas também podem estar associadas à depressão. Este é conhecido como transtorno depressivo induzido por substância/medicamento e de transtorno depressivo devido a outra condição médica (Manual Diagnóstico e Estatístico de Transtornos Mentais, 2014).

O DSM-5 também afirma que os indivíduos têm sintomatologia em comum caracterizada por presença de humor triste, vazio ou irritável, acompanhado de alterações somáticas e cognitivas que irá afetar significativamente a capacidade de funcionamento do indivíduo, o que vai diferenciá-los será os aspectos de duração, momento ou etiologia presumida (DSM-5, 2014).

A depressão é uma doença psicopatológica apontada como uma das patologias com elevada e crescente prevalência na população, representando um sério problema de saúde pública em todo o mundo, caracterizada por inquietação ou retardo psicomotor, humor deprimido durante quase todo o dia, aumento ou diminuição do apetite, significativo ganho ou perda de peso sem realizar dieta, cansaço ou perda de energia, alterações do sono, diminuição da capacidade de pensar e concentrar-se, diminuição acentuada do interesse ou prazer de realizar a maioria ou todas as atividades, sentimentos de inutilidade, culpa exacerbada ou inadequada, confusão e pensamentos de morte recorrentes, ideação suicida sem a elaboração de um plano 
específico, tentativa de suicídio ou elaboração do plano específico para cometê-lo. Além disso, estima-se que até 2030 o transtorno depressivo será a principal causa de incapacidade no mundo inteiro (Alencar et al. 2018).

Segundo Biazus e Ramires (2012) por muito tempo a depressão foi considerada uma psicopatologia apenas da fase adulta, o que vem se modificando pelo aumento de casos entre os adolescentes, apesar da depressão afetar as pessoas em qualquer fase da vida, ela vem atingindo cada vez mais os indivíduos durante a adolescência e o início da vida adulta de uma maneira extremamente preocupante, a incidência da doença entre adolescente varia entre de 3,3 a 12,4\%, com predominância do sexo feminino

Logo, a adolescência é uma fase da vida compreendida entre a infância e o início da vida adulta, marcada por grandes mudanças, além do complexo processo de crescimento e desenvolvimento biopsicossocial, mudanças essas que sofrem influências de diversos fatores, que poderam contribuir para a prevenção ou agravamento de problemas emocionais, como a depressão, (Santana et al. 2020).

A faixa etária de adolescência definida pela Organização Mundial da Saúde (OMS) é de 10 a 19 anos (adolescentes), e a Organização das Nações Unidas (ONU) adota o limite cronológico de 15 a 24 anos (youth), critério este usado principalmente para fins políticos e estatísticos (Ministério da Saúde, 2007). Por outro lado, o Estatuto da Criança e do Adolescente (ECA), considera adolescentes aqueles entre 12 e 18 anos de idade, segundo o Art.2 ${ }^{\circ}$ (Lei n. 8.069, 1990). Sendo assim, o presente trabalho usa como referência de estudo o limite cronológico adotado pelas leis brasileiras, de acordo com o ECA.

Alguns estudos ainda demonstram que todas as pessoas que sofrem de problemas mentais apresentam os primeiros sintomas a partir dos 14 anos, além de apontar que o tratamento precoce entre adolescentes os preveniria de sofrimentos durante toda uma vida e mortes. A Organização Mundial da Saúde, após concluir o primeiro relatório completo acerca dos problemas de saúde entre adolescentes em 109 país, relata que a depressão se tornou a principal causa de enfermidade entre os adolescentes (Ministério da Saúde, 2014).

Os fatores desencadeantes do processo de adoecimento nesses indivíduos são diversos, levando uma parte deles a automutilação, ideação suicidas, tentativas de suicídios, e por fim o próprio suicídio. Segundo a Organização Mundial da Saúde ocorrem cerca de 800.000 mortes por suicídio no mundo, ou seja, uma morte a cada 40 segundos. E no Brasil de 2000 a 2015, ocorreram cerca de 11.947 de mortes causadas por lesões provocadas intencionalmente em indivíduos de 10 a 19 anos, representando cerca de 8,5\% do total desses óbitos, (Cicogna et al. 2019).

Salienta-se ainda que, para garantir o tratamento e acompanhamento multiprofissional desses indivíduos estão disponíveis os Centros de Atenção Psicossocial (CAPS) em suas diferentes modalidades, estes são pontos de atenção estratégicos da Rede de Atenção Psicossocial (RAPS), caracterizada por realizar um conjunto de ações em prol da inclusão e o exercício de direitos de cidadania de usuários e familiares (Lazzari et al. 2019).

De acordo com a lei 10.2016 de 2001, parágrafo único Art. 2 às pessoas portadores de transtornos mentais possuem direito ao melhor acesso de tratamento do sistema de saúde, consentâneo às suas necessidades; devem ser tratadas com humanidade e respeito e no interesse exclusivo de beneficiar sua saúde, visando alcançar sua recuperação pela inserção na família, no trabalho e na comunidade, devem ser protegidas contra qualquer forma de abuso e exploração; deve ser garantindo sigilo nas informações prestadas; possuem direito à presença médica, em qualquer tempo, para esclarecer a necessidade ou não de sua hospitalização involuntária; devem possuir livre acesso aos meios de comunicação disponíveis; devem receber o maior número de informações a respeito de sua doença e de seu tratamento; devem receber tratamento em ambiente terapêutico pelos meios menos invasivos possíveis; devem ser tratadas, preferencialmente, em serviços comunitários de saúde mental.

Deste modo, o presente estudo tem como objetivo geral, identificar os fatores desencadeantes de depressão. Tendo como objetivos específicos: analisar os principais fatores desencadeantes de depressão entre adolescentes; interpretar os resultados apresentados pelos autores; discutir os dados encontrados por categorias. 


\section{Metodologia}

A metodologia adotada trata-se de uma revisão integrativa da literatura, de caráter descritivo, retrospectivo e qualitativo, tendo como foco principal a pesquisa bibliográfica a partir do estudo, identificação e análise do material já publicado para dar subsídio ao tema abordado para o presente trabalho de pesquisa. A revisão Integrativa vem sendo colocada como um importante método para pesquisas no campo da saúde, condensado as pesquisas disponíveis sobre determinado assunto fundamento com fundamento prático e conhecimento científico. A mesma consiste na busca sistemática e rigorosa do processo e análise minuciosa de dados, resultando na diminuição de erros (Santana et al., 2020).

Para obtenção das informações acerca do tema proposto foram realizadas buscas por meio da exploração dos bancos de dados: SciELO (Scientific Electronic Library Online), BVS (Biblioteca Virtual em Saúde) e CAPES (Catálogo de Teses e Dissertações). Fazendo uso dos seguintes descritores: "depressão na adolescência”, "depressão em adolescentes" e “depressão and adolescentes" sendo eles artigos indexados na língua portuguesa.

Utilizando como critérios de inclusão: trabalhos completos na modalidade de artigos científicos, cartilhas e revistas que estão disponíveis na internet e gratuitas acerca do tema proposto, no período de 2010 a 2020.

Os critérios de exclusão foram: produções incompletas de artigos científicos, cartilhas e revistas que não se enquadraram ao tema proposto; produções de anos anteriores a 2010 e superiores a 2020 e que possuíam população menor que 12 anos e maior que 18 anos de idade.

Atualmente os transtornos de humor são constantemente estudados, possuindo uma renovação constante de informações, com isso a pesquisa tem como benefício a compilação de diversos avanços existentes quanto ao tema proposto. Tendo como risco a pesquisa pode não conseguir alcançar todos os avanços nas informações presentes, há uma possibilidade de novidades relacionadas aos transtornos depressivos passarem despercebidos.

As informações coletadas foram retiradas de bancos de dados online, tendo como alvo os avanços nas informações relacionadas aos fatores que desencadeiam a depressão em adolescentes. Para isso, o período estabelecido foi o que possibilitou a identificação dessa informação, compreendendo um período de 10 anos analisado, 2010 a 2020.

Por se tratar de uma pesquisa com utilização de banco de pesquisa com atribuição pública, o trabalho em questão não passou pela avaliação do Comitê de Ética e Pesquisa, mas foram sondados todos os conteúdos utilizados respeitando os princípios éticos estabelecidos na resolução 466/12.

\section{Resultados e Discussão}

Foram identificadas 816 produções nas bases de dados pesquisadas. Destes, 38 foram selecionados para leitura em texto completo, após a leitura, 27 produções foram excluídas por não atenderem ao critério de seleção, restando para a discussão apenas 11 artigos. A predominância dos artigos está localizada nas bases de dados BVS e SCIELO, revistas de enfermagem, revistas de saúde pública, cadernos de saúde coletiva e psicologia, conforme a Tabela 1.

Tabela 1: Quantitativo de artigos encontrados nas bases de dados eletrônicas.

\begin{tabular}{|l|c|c|c|c}
\hline Base de Dados & CAPS & BVS & SCIELO & TOTAL \\
\hline Produção encontrada & 263 & 350 & 203 & 816 \\
\hline Títulos Selecionados & 3 & 4 & 4 & 11 \\
\hline
\end{tabular}

Fonte: Silva e siqueira (2021). 
Para atender o objetivo desta etapa, foi realizada uma leitura exploratória de cunho superficial de todos os materiais, seguida de uma leitura seletiva para escolha do material e por fim o registro das informações extraídas da fonte (numeração, autor/ano, local de publicação, título de estudo e objetivo). Para a segunda etapa foi realizada a análise dos principais fatores desencadeantes de depressão entre adolescentes encontrados nos artigos, interpretação e discussão por categoria os dados encontrados.

Para melhor apresentação, os artigos que atenderam aos critérios de inclusão foram organizados em quadro (Quadro 1) de forma codificada pela sequência alfanumérica, iniciada pela letra D (primeira letra da palavra “depressão") acompanhada por um número arábico em intervalo de 1 a 11, classificados de forma crescente de acordo com os anos de publicação.

Após identificação, análise e interpretação dos dados apresentados pelos autores, os resultados foram divididos por categorias para melhor apresentação da discussão.

Quadro 1: Distribuição dos estudos analisados quanto ao autor/ano, local de publicação, título e objetivo - 2010 a 2020.

\begin{tabular}{|c|c|c|c|c|}
\hline $\mathbf{N}^{\circ}$ & Autor & Local de publicação & Título de estudo & Objeto de estudo \\
\hline D 1 & Ribeiro et al. 2010 & $\begin{array}{l}\text { Psicologia, ciência e } \\
\text { profissão. }\end{array}$ & $\begin{array}{l}\text { Representação Social da Depressão } \\
\text { em uma Instituição de Ensino da } \\
\text { Rede Pública. }\end{array}$ & $\begin{array}{l}\text { Esta pesquisa objetivou apreender as } \\
\text { representações sociais da depressão } \\
\text { de adolescentes do ensino médio, em } \\
\text { uma escola de João Pessoa. }\end{array}$ \\
\hline D2 & Souza et al.2011 & Revista de Salud Pública. & $\begin{array}{l}\text { Desigualdade Social, Delinquência } \\
\text { e Depressão: Um Estudo com } \\
\text { Adolescentes em Conflito com a } \\
\text { Lei. }\end{array}$ & $\begin{array}{l}\text { Avaliar a autopercepção do estado } \\
\text { de saúde de adolescentes em } \\
\text { confronto com a lei, obter } \\
\text { informações relativas ao } \\
\text { relacionamento desses adolescentes } \\
\text { e a instituição destinada a oferecer } \\
\text { atividades socioeducacionais e } \\
\text { propor uma intervenção de } \\
\text { reintegração social. }\end{array}$ \\
\hline D3 & Campos et al. 2014 & $\begin{array}{l}\text { Estudos e Pesquisas em } \\
\text { Psicologia. }\end{array}$ & $\begin{array}{l}\text { Depressão na adolescência: } \\
\text { habilidades sociais e variáveis } \\
\text { sociodemográficas como fatores de } \\
\text { risco/proteção }\end{array}$ & $\begin{array}{l}\text { Este trabalho teve por objetivo } \\
\text { investigar quais dessas variáveis } \\
\text { podem ser fatores de risco ou } \\
\text { proteção da depressão na } \\
\text { adolescência, bem como avaliar o } \\
\text { poder preditivo de um modelo que } \\
\text { inclui essas variáveis. }\end{array}$ \\
\hline D4 & Barbosa et al. 2016 & $\begin{array}{l}\text { Cadernos de Saúde } \\
\text { Coletiva }\end{array}$ & $\begin{array}{l}\text { Sintomas depressivos em } \\
\text { adolescentes em situação de } \\
\text { vulnerabilidade social. }\end{array}$ & $\begin{array}{l}\text { Identificar os principais } \\
\text { indicadores de depressão de } \\
\text { amostra de adolescentes em } \\
\text { vulnerabilidade social e associar } \\
\text { com variáveis } \\
\text { sociodemográficas e } \\
\text { comportamentais. }\end{array}$ \\
\hline D5 & Melo et al. 2017 & $\begin{array}{l}\text { Psicologia: Ciência e } \\
\text { Profissão }\end{array}$ & $\begin{array}{l}\text { Depressão em Adolescentes: } \\
\text { Revisão da Literatura e o Lugar da } \\
\text { Pesquisa Fenomenológica. }\end{array}$ & $\begin{array}{l}\text { Neste artigo, realizamos uma } \\
\text { revisão sistemática da literatura com } \\
\text { o propósito de identificar e discutir } \\
\text { as pesquisas que estão sendo } \\
\text { produzidas no tocante a esse objeto } \\
\text { de estudo. }\end{array}$ \\
\hline D6 & $\begin{array}{l}\text { Fernandes et al. } \\
2017\end{array}$ & Revista de Salud Pública. & $\begin{array}{l}\text { Autoestima, imagem corporal e } \\
\text { depressão de adolescentes em } \\
\text { diferentes estados nutricionais. }\end{array}$ & $\begin{array}{l}\text { Investigar a autoestima, a imagem } \\
\text { corporal e a depressão de } \\
\text { adolescentes em diferentes estados } \\
\text { nutricionais. }\end{array}$ \\
\hline
\end{tabular}




\begin{tabular}{|c|c|c|c|c|}
\hline D7 & Antunes et al. 2018 & $\begin{array}{l}\text { Revista Portuguesa de } \\
\text { Enfermagem de } \\
\text { Saúde Mental, } \\
\text { ESPECIAL. }\end{array}$ & $\begin{array}{l}\text { Regulação emocional e qualidade do } \\
\text { relacionamento com os pais como } \\
\text { preditoras de sintomatologia } \\
\text { depressiva em adolescentes. }\end{array}$ & $\begin{array}{l}\text { Analisar longitudinalmente os } \\
\text { efeitos preditores das estratégias de } \\
\text { regulação emocional e da qualidade } \\
\text { do relacionamento com os pais na } \\
\text { sintomatologia depressiva. }\end{array}$ \\
\hline D8 & Weber et al. 2017 & $\begin{array}{l}\text { International Journal of } \\
\text { Development I and } \\
\text { Educational } \\
\text { Psychology. (INFAD) } \\
\text { Revista de Psicología }\end{array}$ & $\begin{array}{l}\text { Relações entre práticas educativas } \\
\text { parentais percebidas e a autoestima, } \\
\text { sinais de depressão e o uso de } \\
\text { substâncias por adolescentes. }\end{array}$ & $\begin{array}{l}\text { O presente estudo investigou, com } \\
1.400 \text { adolescentes, as relações } \\
\text { existentes entre as práticas estilos } \\
\text { educativos parentais percebidos e } \\
\text { sinais de depressão, autoestima e } \\
\text { comportamentos antissociais de } \\
\text { adolescentes. }\end{array}$ \\
\hline D9 & Costa et al. 2018 & $\begin{array}{l}\text { Revista Portuguesa de } \\
\text { Enfermagem de } \\
\text { Saúde Mental, } \\
\text { ESPECIAL }\end{array}$ & $\begin{array}{l}\text { O efeito moderador da satisfação } \\
\text { com a vida na associação entre a } \\
\text { qualidade da relação pais/filhos(as) } \\
\text { e depressão na adolescência. }\end{array}$ & $\begin{array}{l}\text { Estudar como preditores da } \\
\text { depressão na adolescência a } \\
\text { qualidade da relação pais/filhos(as) e } \\
\text { a satisfação com a vida e explorar o } \\
\text { efeito moderador da satisfação com } \\
\text { a vida, na associação entre a } \\
\text { qualidade da relação entre } \\
\text { pais/filhos(as) avaliadas num } \\
\text { primeiro momento e a depressão na } \\
\text { adolescência avaliada no segundo } \\
\text { momento (seis meses depois). }\end{array}$ \\
\hline D10 & Silva et al. 2019 & $\begin{array}{l}\text { Revista Educação em } \\
\text { Saúde (RESU). }\end{array}$ & $\begin{array}{l}\text { Fatores que influenciam a depressão } \\
\text { na adolescência. }\end{array}$ & $\begin{array}{l}\text { Este trabalho teve por objetivo } \\
\text { elucidar os fatores de risco que } \\
\text { acometem a depressão na } \\
\text { adolescência. }\end{array}$ \\
\hline D11 & Vitti et al. 2020 & Psicologia, Porto Alegre & $\begin{array}{l}\text { Fatores de risco e proteção e } \\
\text { sintomas de depressão na } \\
\text { adolescência. }\end{array}$ & $\begin{array}{l}\text { Avaliou-se a relação entre fatores de } \\
\text { proteção e risco e sintomas } \\
\text { depressivos na adolescência. }\end{array}$ \\
\hline
\end{tabular}

Fonte: Silva e Siqueira (2021).

\subsection{Principais Fatores Desencadeantes de Depressão}

\subsubsection{Relacionamento familiar}

Souza et al (2010) em sua pesquisa, relata que um elevado número de adolescentes referiu pais separados, quase metade mora somente com a mãe e uma proporção semelhante têm a avó como responsável, denotando famílias desestruturadas, com sobrecarga de responsabilidades financeiras e afetivas para mãe ou avó, sendo essa desestrutura familiar um fator de risco para sintomas de depressão, visto que o estudo aponta altos níveis de sintomatologia depressiva nos adolescentes em conflitos com a lei, já que essa desestrutura familiar não afeta somente a saúde psicológica dos jovens, mas também seu caráter diante da sociedade.

Antunes et al. (2017) diz que a depressão envolve dificuldades no nível de regulação emocional, ou seja, os indivíduos revelam dificuldades ao experienciar as emoções, sabe-se que somente ao final da adolescência é que se verifica uma aquisição completa das competências associadas à regulação emocional, o que pode levar a dificultar a regulação cognitiva das emoções, trazendo maior vulnerabilidade ao desenvolvimento de psicopatologia perante acontecimentos de vida negativos. Visto isso, pode-se afirmar que a fase da adolescência por si só já é um fator predisponente à depressão, devido a grande vulnerabilidade que o adolescente apresenta nesse período, e possuir relações conflituosas principalmente com os pais. Quanto às estratégias de regulação emocional (autocrítica; ruminação; catastrofização; culpar o outro; aceitação; reavaliação positiva; planejamento; reorganização positiva; e perspetivar) a pesquisa apontou autocrítica, catastrofização e ruminação como preditoras positivas e significativas de sintomatologia depressiva. Bem como a qualidade do relacionamento com os pais, sugerem que uma maior percepção de conflito nos relacionamentos com os mesmos e uma menor percepção de suporte no relacionamento com a mãe 
demonstram níveis mais elevados de sintomatologia depressiva. Embora seja considerado normativo na adolescência a existência de algum grau de conflito no relacionamento com os pais, situações em que os níveis de conflito são prolongados, intensos e repetidos têm sido associadas a dificuldades no ajustamento psicológico e a maior sintomatologia depressiva.

Segundo os estudos de Costa et al. (2018) os elevados níveis de conflitos com a mãe e níveis baixos de satisfação com a vida (aspectos negativos) funcionam como fatores de risco para o desenvolvimento da sintomatologia depressiva e que diante de uma satisfação boa com a vida (aspectos positivos), estes sintomas tendem a ser amenos, funcionando como fator de proteção, mesmo havendo relações conflituosas no ambiente familiar, ou seja, mesmo perante a má qualidade da relação familiar existem fatores que promovem saúde mental.

Weber (2017) diante de seu estudo aponta que 75,5\% dos adolescentes com indícios de depressão possuem pais negligentes, ou seja, pais que não colocam regras e não demonstram responsabilidade diante da criação de seus filhos, o que por sua vez, faz o adolescente se sentir abandonado, gerando raiva, tristeza, solidão e culpa, corroborando para o aparecimento de sinais depressivo e comportamentos disruptivos, tais como o uso de drogas e bebida alcoólica. Pode-se confirmar essa relação diante da pesquisa apresentada, que correlaciona o uso de drogas à depressão, onde 30,4\% dos indivíduos com sinais de depressão fazem uso de substâncias ilícitas e possuem pais negligentes.

Silva et al. (2017) concluiu que o mau relacionamento familiar (principalmente menor suporte materno) e a situação de vulnerabilidade são os principais fatores que levam à depressão. Outro fator observado foi a questão emocional e também o grupo com maior incidência são as mulheres devido a coerção social, autocrítica e dificuldade de aceitação. Por outro lado, quando se leva em consideração a depressão associada ao uso de álcool e drogas a prevalência é maior no sexo masculino, que também advém de relacionamentos conflituosos com os pais.

\subsubsection{Vulnerabilidade social}

Melo et al. (2017) descreve em seus estudos que houve um consenso entre os autores na classificação de determinadas variáveis como fatores de risco ou proteção para o desenvolvimento da sintomatologia depressiva como vulnerabilidade social, no que concerne à vulnerabilidade social, os autores constataram que a prevalência de depressão apresenta-se em indivíduos de classes sociais mais baixas, pois além das privações advindas da baixa renda, coexistem às dificuldades do cotidiano como desemprego, exposição a violência nas ruas e em algumas situações casos violência doméstica, uniões precoces e transitórias, alcoolismo e o uso de drogas.

Barbosa et al. (2016) como resultado de suas pesquisas observou a alta prevalência de sintomatologia depressiva em adolescentes em situação de vulnerabilidade social, onde constatou que diante das variáveis "perda de peso ou ganho de peso", "baixa autoestima" e "fadiga" atribuíram para dar poder de explicação à 20,9\% da depressão infantil de adolescentes em situação de vulnerabilidade social, tendo como prevalência o sexo masculino, apresentando 2,24 maior probabilidade de desenvolver a depressão em relação as meninas, os autores atribuem esse fato devido a vulnerabilidade social, bem como ao possível risco de associação com drogas.

\subsubsection{Gênero e idade}

De acordo com os estudos de Campos et al. (2014) ser do sexo feminino, ter idade superior a 12 anos de idade e apresentar déficits ou dificuldade em relação as habilidades de civilidade (cumprimentar, capacidade de agradecer ou solicitar um favor) são fatores de risco para o desenvolvimento da depressão, essa baixa frequência de habilidades sociais acaba por favorecer a rejeição dos colegas, aumentando a probabilidade de ser excluído de grupos, gerando sentimentos de baixa autoestima, o que corrobora para o aparecimento de sintomas depressivos. 
Corroborando com esses estudos Vitti et al. (2020) constatou em suas pesquisas que as mulheres comparadas aos homens mostram maior vulnerabilidade frente à depressão, porém mesmo com o surgimento precoce dos sinais depressivos, a cronicidade apresenta-se de modo semelhante para ambos os gêneros. Podendo ser justificada devido às mudanças biopsicossociais inerentes a adolescência, como as questões relacionadas à puberdade, sabe-se que para as meninas as mudanças são muito mais significativas, não só devido às mudanças físicas, mas as biológicas. Evidenciou-se também que em relação à idade, as diferenças de gênero quanto à sintomatologia depressiva acentuaram-se entre os adolescentes com 16 anos, por outro lado, quanto maior o nível de desamparo, desesperança e solidão maiores são as chances de desenvolver sintomatologia depressiva.

Em contrapartida, Barbosa et al. (2016) em seu estudo sobre jovens em situação de vulnerabilidade social, constatou que o sexo masculino apresentou duas vezes maior probabilidade de desenvolver depressão em relação as meninas, os autores atribuem esse fato devido a vulnerabilidade social, bem como ao possível risco de associação com drogas.

\subsubsection{Insatisfação corporal}

Fernandes et al. (2017) apontou evidências empíricas de que a depressão está relacionada negativamente com a autoestima e insatisfação corporal, e verificou também que de modo geral as meninas possuem níveis superiores de depressão comparada aos meninos. Vale ressaltar que não se sabe ao certo o porquê das mulheres apresentarem níveis mais elevados de depressão, porém algumas pesquisas apontam para as seguintes questões: motivos psicossociais como, menor status social, regras diferenciada para homens e mulheres, maior ocorrência de eventos estressantes, corporal e biológicos como mudanças hormonais, gravidez e neurotransmissores, favorecem a maior depressão entre as mulheres. Corroborando com esta pesquisa, Santos et al. (2019) em seus estudos confirmou majoritariamente a relação da imagem corporal com a depressão, afirmando que o modo como este transtorno aumenta a vulnerabilidade entre o real e o percebido, elevando a autocrítica, assim agravando os sintomas depressivos, dificultando a auto aceitação.

\subsubsection{Aspectos psicoafetivos e psicossociais}

Ribeiro et al. (2010) aponta que a tristeza, desilusão amorosa, dificuldade de relacionamento social, carência afetiva, solidão juntamente com a ideação suicida são fatores associados à depressão. Também relata que a dificuldade de relacionar corrobora com o surgimento da depressão pois o adolescente, ao não se adaptar ao meio por dificuldades familiares, problemas relacionados à puberdade e término de relacionamento, tende ao isolamento, apresentando sentimentos de inferioridade e de baixa autoestima. Visando que o relacionamento social tem papel importante no desenvolvimento na vida biopsicossocial desse jovem. Corroborando com esta pesquisa, Silveira et al. (2012) em seus estudos diz que a depressão está ancorada aos aspectos psicoafetivos, pois o sentimento de tristeza está presente como principal eixo da natureza afetiva e psicológica, além do desânimo, pensamentos suicidas, sentimentos negativos e traumas. Demonstrando que os problemas afetivos são os principais motivos para a procura de ajuda psicológica.

\section{Considerações Finais}

O presente artigo alcançou seus objetivos pois encontrou as respostas relacionadas à quais seriam os principais fatores desencadeantes de depressão entre adolescentes, evidenciando que a depressão é multifacetada, ou seja, seu desenvolvimento advém de vários fatores, além de enfatizar que a adolescência por si só já é uma fase complexa. Nesta fase os adolescentes lidam com várias situações relacionadas ao seu desenvolvimento biopsicossocial como, a luta por aceitação na sociedade e nos grupos de amigos, relacionamentos amorosos conturbados, baixa autoestima e problemas internos. A somatização desses fatores acompanhadas de conflitos familiares, pais negligentes e falta de regras, percebe-se que não há a construção de um vínculo com 
esses jovens o que seria um fator protetivo, favorecendo o aumentando das chances do desenvolvimento da patologia.

Durante as pesquisas e análise dos dados, diversos estudos mostraram a existência de um elo entre a relação familiar e o surgimento da depressão, pois é no convívio familiar que o indivíduo busca proteção, tranquilidade e apoio frente às adversidades da vida. Assim, ficou evidente que o contexto familiar é primordial para o desenvolvimento do indivíduo pois é ele que vai proporcionar segurança frente a situações percebidas com mais intensidade nesse momento, quando esse ambiente ou a relação com os pais não traz essa segurança fortalecendo o vínculo de confiança, tem-se como resposta adolescentes com manifestações de sintomas depressivas, pois eles sofrem sozinhos, sem um suporte adequado para lidar com a fase do adolescer.

Por se perceber que o contexto familiar é de grande relevância para o desencadeamento de sintomas depressivos, é imprescindível a busca por ações que possam melhorar o relacionamento familiar de forma geral, e em especial o favorecimento de vínculo entre pais e filhos, é por meio desse relacionamento que o indivíduo vai começar a ter base para o seu desenvolvimento, é o primeiro lugar de busca por suporte e acolhimento frente a todos os problemas encontrados.

Assim, o presente estudo ajudará a compilar conhecimentos para outros estudantes e profissionais acerca dos fatores desencadeantes de depressão entre adolescente, fornecendo maior conhecimento acima da psicopatologia, o que possibilitará trabalhar mais as questões da prevenção dos transtornos depressivos. Dessa forma, é necessário trabalhar mais as políticas de saúde mental acerca da prevenção e tratamento precoce da doença, além de buscar meios de intervir junto dos adolescentes e dos pais, na intenção de melhorar o relacionamento entre eles.

Para trabalhos futuros seria de suma importância realizar pesquisas acerca da implementação de políticas de saúde relacionadas ao programa do adolescente (PROSAD) que visa o cuidado integral da saúde do adolescente, na busca de promover uma melhor assistência para esses adolescentes,

\section{Referências}

Alencar, A. V. M., M, T. L. G., Rivalina, Fernandes, M. M., \& Rodrigues, M. S. (2018). A relação entre depressão e ideação suicida na juventude. Id on Line Revista Multidisciplinar e de Psicologia, 12(39). https://idonline.emnuvens.com.br/id/article/download/1011/1450

Apa. (2014). Manual diagnóstico e estatístico de transtornos mentais. (5 ${ }^{a}$ ed.). American Psychiatric Association Porto Alegre: Artmed. http://www.niip.com.br/wp-content/uploads/2018/06/Manual-Diagnosico-e-Estatistico-de-Transtornos-Mentais-DSM-5-1-pdf.pdf

Antunes, J., Matos, A. P., Costa, J. J. (2018). Regulação emocional e qualidade do relacionamento com os pais como preditoras de sintomatologia depressiva em adolescentes. Revista Portuguesa de Enfermagem de Saúde Mental, ESPECIAL. http://dx.doi.org/10.19131/rpesm.0213

Barbosa, D. G., et al. (2016). Sintomas depressivos em adolescentes em situação de vulnerabilidade social. Cadernos de saúde coletiva, 24 (2), pp. 221-227, Rio de Janeiro. https://doi.org/10.1590/1414-462X201600020195

Biazus C. B., \& Ramires, V. R. R. (2012). Depressão na adolescência: uma problemática dos vínculos. Psicologia em Estudo, Maringá, 17 (1), pp. 83-91 https://doi.org/10.1590/S1413-73722012000100010

Brasil (2007). Ministério da Saúde. Secretaria de Atenção à Saúde. Área de Saúde do Adolescente e do Jovem. Marco legal: saúde, um direito de adolescentes. Brasília : Editora do Ministério da Saúde, ed. 10. https://bvsms.saude.gov.br/bvs/publicacoes/07_0400

Brasil (2019). Ministério da Saúde. Secretaria de Atenção Primária à Saúde. https://aps.saude.gov.br/noticia/5758

BRASIL (2021). Ministério da Mulher, da Família e dos Direitos Humanos. Lei no 8.069, 13 de julho de 1990 . Dispõe sobre o Estatuto da Criança e do Adolescente e dá outras providências. Diário Oficial da União.https://www.gov.br/mdh/pt-br/assuntos/noticias/2021/julho/trinta-e-um-anos-do-estatuto-dacrianca-e-do-adolescente-confira-as-novas-acoes-para-fortalecer-o-eca/ECA2021_Digital.pdf

Campos, J. R, Del Prette A., Del Prette Z. A. P. (2014). Depressão na adolescência: habilidades sociais e variáveis sociodemográficas como fatores de risco/proteção. Estudos e Pesquisa em Psicologia, 14 (2) ,pp. 408-428, Rio de Janeiro. http://pepsic.bvsalud.org/pdf/epp/v14n2/v14n2a03

Cicogna, J. I. R., Hillesheim D., Halla, A. L. L. C. (2019). Mortalidade por suicídio de adolescentes no Brasil: tendência temporal de crescimento entre 2000 e 2015. Jornal Brasileiro de Psiquiatria, 68 (1). https://doi.org/10.1590/0047-

Costa, B. S., Matos, A. P., Costa. J. J. (2016). $O$ efeito moderador da satisfação com a vida na associação entre a qualidade da relação pais/filhos(as) e depressão na adolescência. Revista Portuguesa de Enfermagem de Saúde Mental, ESPECIAL 6. http://dx.doi.org/10.19131/rpesm.0212

Fernandes, A. R., et al. (2017). Autoestima, imagem corporal e depressão de adolescentes em diferentes estados nutricionais. Revista de Salud Pública. https://doi.org/10.15446/rsap.v19n1.47697 
Research, Society and Development, v. 10, n. 16, e432101624295, 2021 (CC BY 4.0) | ISSN 2525-3409 | DOI: http://dx.doi.org/10.33448/rsd-v10i16.24295

Feitosa, D. A. M. M., Bohry, S., Machado, E. R. (2011). Depressão: família e seu papel no tratamento do paciente. Encontro: Revista de Psicologia, 14,(21), p.127-144. https://seer.pgsskroton.com/renc/issue/view/236

Lazzari, A. P., et al. (2019). Depressão em usuários que frequentam o caps. Anuário Pesquisa e Extensão Unoesc São Miguel do Oeste, 4. https://portalperiodicos.unoesc.edu.br/apeusmo/article/view/20460

Lei 10.216 de 2001 (2014). Reforma Psiquiátrica e os Direitos das Pessoas com Transtornos Mentais no Brasil. Centro de Educação em Direitos Humanos. https://saudedireito.org/2014/05/26/lei-10-216-de-2001-reforma-psiquiatica-e-os-direitos-daspessoas-com-transtornos-mentais-no-brasil/

Melo, A. K., Siebra, E. J., Moreira, V. (2017). Depressão em Adolescentes: Revisão da Literatura e o Lugar da Pesquisa Fenomenológica. Psicologia: Ciência e Profissão, 37 (1), p. 18-34. https://doi.org/10.1590/1982-37030001712014

Ribeiro, K. C. S., et al. (2010). Representação Social da Depressão em uma Instituição de Ensino da Rede Pública. Psicologia, ciência e profissão, 30 (3), p. 448-463. https://doi.org/10.1590/S1414-98932010000300002

Santana, S. K., Bueno, S. B., Pompermaier, C. (2020). Depressão e suicídio na adolescência: uma revisão de literatura. Anuário Pesquisa e Extensão Unoesc Xanxerê 5. https://unoesc.emnuvens.com.br/apeux/article/view/24269

Santos, A. J. N., Souza, G. S., Galvão, P. V. M. (2019). Depressão em adolescentes e sua relação com a imagem corporal. Multidisciplinary reviews. DOI: $10.29327 /$ multi.2019001

Silva, D. O., et al. (2019). Fatores que influenciam a depressão na adolescência. Revista Educação em Saúde (RESU),7 (1). http://periodicos.unievangelica.edu.br/index.php/educacaoemsaude/article/view/3791

Silveira, K. C. R., et al. (2012). Representações sociais e sofrimento psíquico de adolescentes com sintomatologia depressiva. Psicologia: Teoria e Prática,14 (3), p. 18-33. http://www.redalyc.org/articulo.oa?id=193824911002

Souza, E. M., Silva-Abrão, F. P., Oliveira-Almeida, J. (2011). Desigualdade Social, Delinquência e Depressão: Um Estudo com Adolescentes em Conflito com a Lei. Revista de Salud Pública 13 (1), p.13-26. http://www.scielo.org.co/scielo.php?script=sci_arttext\&pid=S0124-00642011000100002

UNA-SUS (2014). Ministério da Saúde. Secretaria de Gestão do Trabalho e da Educação na Saúde. https://www.unasus.gov.br/noticia/oms-adverte-quedepressao-e-doenca-mais-frequente-na-adolescencia

Vicelli, L. D. (2015). Depressão e suas diversas possibilidades de tratamento disponíveis nas Unidades Básicas de Saúde. Monografia (Especialização), Universidade Federal de Santa Catarina, Florianópolis pp. 1-28. https://ares.unasus.gov.br/acervo/html/ARES/13152/1/Leandro_Donato_Vicelli.pdf

Vitti, L. S., Faro, A., BAPTISTA, M. N. (2020). Fatores de risco e proteção e sintomas de depressão na adolescência. Psicologia, Porto Alegre 51 (4), p. 1-11. https://doi.org/10.15448/1980-8623.2020.4.34353

Weber, L.N. D. (2017). Relações entre práticas educativas parentais percebidas e a autoestima, sinais de depressão e o uso de substâncias por adoles centes International Journal of Development I and Educational Psychology. (INFAD) Revista de Psicología, 1 - monográfico 1, p. 157-168 .https://doi.org/10.17060/ijodaep.2017.n1.v2.928 